\title{
Commentary Accelerated partial breast irradiation: technically feasible but who will benefit?

\author{
Gillian Ross
}

Royal Marsden Hospital and Institute of Cancer Research, London, UK

Corresponding author: Gillian Ross, Gillian.ross@rmh.nhs.uk

Published: 5 April 2005

This article is online at http://breast-cancer-research.com/content/7/3/110

Breast Cancer Research 2005, 7:110-112 (DOI 10.1186/bcr1016)

(c) 2005 BioMed Central Ltd

See related commentary by Keisch, page 106 [http://breast-cancer-research.com/content/7/3/106]

\begin{abstract}
Modern breast cancer radiotherapy aims to increase uncomplicated cure rates. A priority is reduction of late effects which include chronic chest wall or breast pain, poor cosmesis, and cardiac toxicity. As breast screening detects early cancers we may be able to safely restrict irradiation postlumpectomy to the tumour bed with a margin, defining a 'partial breast' target volume for treatment. Differing technical approaches to partial breast irradiation are being evaluated in phase III studies with standard whole breast irradiation. These include intra-operative single doses, hypo-fractionated accelerated brachytherapy, and LINAC (linear accelerator)-based three-dimensional external beam therapy.
\end{abstract}

\section{Introduction}

Breast conservation has become the standard of care for women with small cancers who wish to avoid mastectomy. During the past 20 years carefully conducted clinical studies have demonstrated the efficacy of lumpectomy followed by radiotherapy in achieving survival levels equivalent to those with mastectomy, with major improvements in body image and psychosexual functioning [1]. The price of such progress has not been cheap, because the change in practice has been a major resource issue for many oncology services where health care planning failed to predict the demanding (and appropriate) increase in use of radiotherapy for most common cancers.

The key issue, then, remains how do we maintain excellent breast conservation rates after lumpectomy, while reducing the morbidity of whole breast radiotherapy (WBRT)? Longterm breast or chest wall pain and poor cosmesis in a relatively small proportion of patients has always been considered by the majority of women to be an acceptable trade-off against preservation of the breast. More serious concerns regarding long-term efficacy emerged from the Early Breast Trialists overview [2], which confirmed excess cardiac events in left-sided breast cancers.

One approach to reducing late radiotherapy morbidity with WBRT has been to seek to define those patients with small, node-negative cancers, who are most likely to have been 'cured' by lumpectomy alone! This philosophy originally gained support from randomized trial evidence indicating that there was no apparent difference in survival rates after lumpectomy with or without radiotherapy. Recent publications support the notion that WBRT after lumpectomy produces excellent local control in around $96 \%$ of patients at 5 years, with no difference in risk for distant metastases or survival. Questions have now been raised regarding the level of benefit from WBRT in women older than 70 years, and in those with tumours $<2 \mathrm{~cm}$ that are oestrogen receptor positive and node negative, although it is clear that 5 years of follow up is not adequate for evaluating overall efficacy [3].

Even with careful case selection it is likely that with continued follow up local failures after lumpectomy alone will continue to rise. Keisch [4] cited the Milan QUART (Quadrantectomy, Axillary Dissection and Radiotherapy) study [5], which found evidence that patients receiving generous 'quadrantic' surgery still enjoy further benefit from radiotherapy with sufficient follow up. However, another interpretation of the study is that nonsurgical quadrant therapies (i.e. intraoperative radiotherapy [IORT]) are equally likely to prejudice local control rates with sufficient follow up, unless cases are so well selected that they are likely to have already been cured by their surgery. As the average life expectancy of women approaches 80 years in most Western countries, even selecting an older cohort to omit WBRT may have an 
impact on future breast conservation rates and possibly on overall disease-free survival.

A much more challenging, alternative approach to selective avoidance of WBRT has been to refine adjuvant breast radiotherapy techniques. A number of investigators are actively reexploring tumour bed irradiation (partial breast radiotherapy [PBRT]) combined with accelerated, hypofractionated therapy. We should remember that the philosophy of PBRT was previously explored and discarded when interstitial implants and electron beam approaches failed to generate adequate local control rates with acceptable cosmesis [6,7]. However, several key clinical factors and technical advances now drive an exciting reappraisal of how best to use adjuvant radiotherapy in early breast cancer. These include an increasing proportion of women with early and in situ breast cancer, an awareness of the potential for late cardiac toxicity, and the health economics of protracted treatment courses with significant equipment and human resource needs. Are faster, fewer fractions delivered to partial breast volumes the solution?

\section{How can we localize the tumour cavity after lumpectomy?}

As a result of breast screening, we are seeing increased numbers of small cancers with better prognosis. In some centres ductal carcinoma in situ constitutes $20-30 \%$ of all new cancers. With increasingly compelling evidence that the main effect of WBRT is to reduce significantly local recurrence in the index quadrant, without a measurable impact on subclinical foci in other sectors, we need to redefine the clinical target volume (CTV) after lumpectomy.

One of the principle concerns in defining practice for partial breast irradiation (PBI) is lack of experience in defining a CTV after lumpectomy. This is compounded by the fact that the surgery is not performed in the plane of the breast ductal system, creating potential to have varying three-dimensional clearance following tumourectomy. Surgical clips appear to be the current 'gold standard' for defining the resection margins, but the frequency and extent of clip migration have yet to be fully defined, posing problems for external beam PBRT in particular.

\section{What is the appropriate margin for a clinical target volume?}

The appropriate CTV for PBI is subject to considerable debate; clinicians have concluded empirically that defining a treatment margin of 10-20 mm should encompass any microscopic margins in the 'majority' of patients; this will need to be proven to be the standard of practice in this area in prospective studies, in which close attention is paid to documentation of surgical margins. Concern over the need to avoid skin in the high-dose region, with potential for necrosis or telangectasia, have led to empirical adoption of $5 \mathrm{~mm}$ clearance from CTV to skin, resulting in the potential for inadequate anterior margin therapy without careful case selection.

\section{Can we treat partial breast irradiation volumes reproducibly?}

In addition to reservations regarding the definition of optimal planning target volume in accelerated partial breast irradiation (APBI), a further concern relates to reproducibility and verification of fractionated courses. Clearly, this is mostly a concern for external beam PBI rather than brachytherapybased PBI. In their study, Frazier and coworkers [8] analyzed anterior-posterior motion of medial and lateral field borders between normal inhalation and exhalation using computed tomography coupled with automatic breathing control. Movement ranged from 0.6 to $0.9 \mathrm{~cm}$ on these borders. In a more recent study of target motion using surgical clips to define the lumpectomy cavity in seven cases [8] the maximal clip displacement with respiration ranged from 3 to $9 \mathrm{~mm}$, with a median of $6 \mathrm{~mm}$. The investigators concluded that symmetrically expanding the free-breathing CTV by $5 \mathrm{~mm}$ would be sufficient to account for target motion in most patients. It proved more difficult to allow for additional elements of geometrical uncertainty (e.g. patient position between fractions), and they concluded that a CTV to planning target volume margin of $10 \mathrm{~mm}$ would be more likely to guarantee target coverage. Although issues remain regarding reproducibility, a major limitation of this approach remains the significant dosimetry and LINAC (linear accelerator) resources required to deliver fractionated external beam-based conformal PBI, and constraints required to achieve acceptable lung, cardiac and contralateral breast doses. Although intensity-modulated radiotherapy has produced significant improvements in dose delivery in several tumour types, it remains far from clear that it can improve uncomplicated breast cancer local control, largely because of concerns over increased contralateral breast dose with multiple beam delivery around the thorax. If reduction in unnecessary cardiac dosing were the sole concern, then 'mini-tangent' conventional beams avoiding the lower breast pole or incorporation of cardiac beam shaping would achieve the objective in upper pole cancers very simply [9].

\section{Partial breast irradiation is feasible, but is it a major therapeutic advance? Is biology the key?}

Techniques for APBI include LINAC-based three-dimensional conformal external beam therapy [10], brachytherapy [11], and intraoperative, single fraction treatment. The latter two approaches are being evaluated in two randomized studies against WBRT in Europe.

The European Institute of Oncology reported results of a phase I/II trial in which patients with tumours $<2.5 \mathrm{~cm}$ $(n=84)$ were treated with electron beam IORT alone after lumpectomy with a $1 \mathrm{~cm}$ margin [12]. A PTV of $1-3 \mathrm{~cm}$ around the clinical tumour bed was chosen. Doses were escalated from 17 to $21 \mathrm{~Gy}$. Acute toxicity was minimal, with grade $1-2$ fibrosis occurring in 5\% receiving 21 Gy. It was concluded that the technique is feasible; it is being evaluated in a randomized study against WBRT. A similar study 
conducted at University College Hospital, London employed IORT with soft $X$-rays at the time of lumpectomy [13]. This system uses a miniature electron beam-driven $\mathrm{X}$-ray source called INTRABEAM $(\mathrm{PeC})$ that emits soft $X$-rays $(50 \mathrm{kV})$ from within the breast. The $\mathrm{X}$-rays are emitted from the tip of a $10 \mathrm{~cm} \times 3.2 \mathrm{~mm}$ diameter probe, which is enclosed in a spherical applicator (available in $2.5-5 \mathrm{~cm}$ diameter sizes), which in turn is inserted in the tumour bed and IORT is delivered over about $25 \mathrm{~min}$. The prescribed dose is 5 and 20 Gy at $1 \mathrm{~cm}$ and $0.2 \mathrm{~cm}$, respectively, from the tumour bed. The biologically effective dose is calculated to be 7-53 Gy for $\alpha / \beta=10$ and $20-120$ Gy for $\alpha / \beta=1.5$. Quick attenuation of radiation reduces the damage to normal tissues and allows radiotherapy to be delivered in a standard operating theatre.

Concerns have been raised that the biological equivalent dose for these IORT systems may be too low for tumour effect and too 'hot' for normal tissues. Further criticisms of these techniques include lack of knowledge of resection margins or node status before therapy. Most importantly, we currently know little of the biological effect of large single, high dose rate treatments on microscopic breast cancer, and the current study designs will not elucidate the optimal dosing.

Keisch [4] cited recently published studies of APBI with median follow up of at least 5 years. Although local recurrence rates in two of these studies of $1.2-2.0 \%$ are very promising, a third study, with a recurrence rate of $4.4 \%$, might be regarded as less satisfactory. A recent study of lumpectomy with or without WBRT, conducted in women older than 70 years with tumour size $<2 \mathrm{~cm}$ and oestrogen receptor positive, yielded local recurrence rates of $4 \%$ without any radiotherapy, which were reduced significantly to $1 \%$ with WBRT. This raised questions regarding the need for any adjuvant radiotherapy in carefully selected subgroups of patients with cancers of good prognosis. Clinicians must bear in mind that for many women avoidance of local recurrence of breast cancer, even if manageable by mastectomy, is paramount. In a recent study conducted by Fyles and coworkers [14], 899 out of 1572 patients (57\%) refused randomization after lumpectomy to radiotherapy or tamoxifen alone. Hence, future randomized studies of WBRT versus APBI will need to demonstrate greater efficacy than surveillance with anti-oestrogen treatment in this particular group.

\section{Conclusion}

The PBI approach offers potentially the most significant advance since breast conservation became the standard of care. It seems highly likely that future generations of women with early breast cancer will receive focal tumour bed irradiation, with excellent breast conservation rates and improved uncomplicated cure rates. A major factor in favour of APBI currently appears to be patient convenience, and it is done within the context of well designed, randomized clinical trials, in which standard whole breast irradiation techniques are compared with more innovative tests of target size definition and fractionation. We must still define those patient groups most likely to benefit from PBI. Premature and empirical adoption of the technology without the results of such studies is likely to set back the entire field.

\section{Competing interests}

The author(s) declare that they have no competing interests.

\section{References}

1. Morrow M, Harris JR: Local management of invasive breast cancer. In Diseases of the Breast, 2nd ed. Edited by Harris JR, Lippman ME, Morrow M, Osborne CK. Philadelphia: Lippincott Williams \& Williams; 2000:515-561.

2. Early Breast Cancer Trialists' Collaborative Group: Favourable and unfavourable effects on long-term survival of radiotherapy for early breast cancer: an overview of the randomised trials. Lancet 2000, 355:1757-1770.

3. Hughes KS, Schnaper LA, Berry D, Cirrincione C, McCormick B, Shank B, Wheeler J, Champion LA, Smith TJ, Smith BL, et al:: Lumpectomy plus tamoxifen with or without irradiation in women 70 years of age or older with early breast cancer. $N$ Engl J Med 2004; 351:971-7.

4. Keisch $\mathrm{M}$ : Accelerated partial breast irradiation: the case for current use. Breast Cancer Res 2005, 7:106-109.

5. Veronesi U, Cascinelli N, Mariani L, Greco M, Saccozzi R, Luini A, Aguilar M, Marubini E: Twenty-year follow-up of a randomized study comparing breast-conserving surgery with radical mastectomy for early breast cancer. N Engl J Med 2002, 347:12271232.

6. Fentiman IS, Poole C, Tong D, Winter PJ, Mayles HM, Turner P, Chaudary MA, Rubens RD: Iridium implant treatment without external radiotherapy for operable breast cancer. A pilot study. Eur J Cancer 1991, 27:447-450.

7. Ribeiro GG, Magee B, Swindell R, Harris M, Banerjee SS: The Christie Hospital Breast Conservation Trial: an update at $\mathbf{8}$ years from inception. Clin Oncol 1993, 5:278-283.

8. Frazier RC, Vincini FA, Sharpe MB, Fayad J, Yan D, Maertinez AA, Wong JW: The impact of respiration on whole breast radiotherapy: a dosimetric analysis using active breathing control [abstract]. Int J Radiat Oncol Biol Phys 2000, 48(Suppl 1):200.

9. Landau D, Adams EJ, Webb S, Ross G: Cardiac avoidance in breast radiotherapy: a comparison of simple shielding techniques with intensity-modulated radiotherapy. Radiother Oncol 2001, 60:247-255.

10. Baglan KL, Sharpe MB, Jaffray D, Frazier RC, Fayad J, Kestin LL Remouchamps V, Martinez AA, Wong J, Vicini FA: Accelerated partial breast irradiation using 3D conformal radiation therapy (3D-CRT). Int J Radiat Oncol Biol Phys 2003, 55:302-311.

11. Keisch M, Vicini F, Kuske RR, Hebert M, White J, Quiet C, Arthur $D$, Scroggins T, Streeter O: Initial clinical experience with the MammoSite breast brachytherapy applicator in women with early-stage breast cancer treated with breast-conserving therapy. Int J Radiat Oncol Biol Phys 2003, 55:289-293.

12. Veronesi U, Orecchia R, Luini A, Gatti G, Intra M, Zurrida S, Ivaldi $G$, Tosi G, Ciocca M, Tosoni A, et al.: A preliminary report of intra-operative radiotherapy (IORT) in limited-stage breast cancers that are conservatively treated. Eur J Cancer 2001, 37:2178-2183.

13. Vaidya JS, Baum M, Tobias JS, D'Souza DP, Naidu SV, Morgan S, Metaxas M, Harte KJ, Sliski AP, Thomson E: Targeted intra-operative radiotherapy (Targit): an innovative method of treatment for early breast cancer. Ann Oncol 2001, 12:1075-1080.

14. Fyles AW, McCready DR, Manchul LA, Trudeau ME, Merante $P$, Pintilie M, Weir LM, Olivotto IA: Tamoxifen with or without breast irradiation in women $\mathbf{5 0}$ years of age or older with early breast cancer. N Engl J Med 2004, 351:963-970. 